\title{
Crisis en la infancia: ¿qué piensan, sienten y dicen los niños sobre la separación de sus padres?
}

\author{
Crisis in the infancy: what the children think, feel \\ and say, on the separation of the parents?
}

Recibido: enero 11 de 2010 | Revisado: agosto 12 de 2010 | Aceptado: noviembre 14 de 2010

\author{
Oriana Cifuentes Neumann ${ }^{* *}$ \\ NeVA MiliciC MÜlLER ${ }^{* * *}$ \\ Pontificia Universidad Católica de Valparaiso, Chile
}

SICI: 1697-9267(201206)11:2<469:CIQPSD>2.0.TX;2-R

Para citar este artículo. Cifuentes, O. \& Milicic, N. (2012). Crisis en la infancia: iqué piensan, sienten y dicen los niños sobre la separación de sus padres? Universitas Psychologica, 11(2), 469-480.

Departamento de Psicología Social, Facultad de Psicología, Passeig Vall d'Hebron, 171, 08035 Barcelona, España. E-mails: ritaberger@ub.edu, myepes@ub.edu,sdiazdequijano@ub.edu

** Psicóloga Infanto-juvenil. Especialista en Psicoterapia Sistémica en niños y adolescentes. Magíster en Psicología Clínica, U.C. E-mail: orianacifuentes@ yahoo.com. ResearcherID: Cifuentes, O. F-36512012.

**** Doctora en Psicología. Magíster en Educación. Profesora titular de la Pontificia Universidad Católica de Chile.E-mail: neva.milicic@gmail.com

\section{RESUMEN}

El artículo presenta el estudio cualitativo de narrativas de niños de 9 a 12 años de edad respecto de la separación de sus padres. La muestra estuvo constituida por 12 niños de nivel socioeconómico medio y el análisis de los datos se desarrolló según los procedimientos de la escuela Grounded Theory. El propósito del estudio es evidenciar la experiencia de niños chilenos respecto a la separación de sus padres y el significado que dicha experiencia tiene actualmente en sus vidas. Los resultados del estudio indican que la separación de los padres constituye una crisis para los niños, quienes la representan como la pérdida de la familia nuclear. Esta vivencia afecta su desempeño conductual y socioemocional, generándose cambios de estado de ánimo que se evidencien en el ámbito escolar y social. Transcurridos dos o tres años, los niños logran tomar cierta distancia de la experiencia y evaluarla desde una nueva perspectiva. Es así como algunos de ellos resignifican la crisis considerando elementos favorables que identifican en el presente. Palabras clave autores

Separación de los padres, crisis, narrativa infantil.

Palabras clave descriptores

Investigación cualitativa, Psicología del desarrollo, afrontamiento, infancia.

\section{A B S T R A C T}

The article presents the qualitative study of narratives of children from 9 to 12 years old, about the separation (divorce) of their parents. The sample consisted of 12 children (boys/girls), middle class and the analysis of the data was done according the procedures of the "Grounded Theory". The purpose of the study is to reveal the experience of Chilean children about their parents divorce and the meaning of that experience in their lives now. The study results indicate that the parental separation (divorce) is a crisis for the child, who represents it as the lost of the nuclear family. This experience affects his behavioral and socioemocional performance generating mood changes that are evident in the school and social area. After two or three years, the child manage to take some distance from the experience and evaluate it from a new perspective. Thus some of them reframing the crisis considering favorable elements identified in the present.

Key words authors

Parental separation (divorce), crisis, children narrative.

Key words plus

Qualitative research, development psychology, coping, childhood. 


\section{Antecedentes}

\section{La experiencia de separación de los padres y el impacto en el desarrollo infantil}

La separación de los padres ha sido descrita por algunos autores como una de las experiencias más difíciles y dolorosa de enfrentar para los hijos. La familia constituye el soporte psicológico, físico y emocional del niño hacia la madurez. Con la separación de los padres, la estructura familiar previa se modifica provocando en los niños ${ }^{1}$ sentimientos de soledad y temor respecto de lo que ocurrirá en su cotidianeidad y en el futuro (Capponi, 2003).

Wallerstein y Blakeslee (1996) señalan, respecto de la separación de los padres, que lo que resulta bueno para los adultos no necesariamente es favorable para los niños. Los niños poseen sus propias creencias, sentimientos e ideas respecto de la separación de sus padres. Así, las circunstancias que enriquecen la vida del adulto luego de la separación, impone cambios y pérdidas significativas que los niños deben enfrentar. De ahí que la separación interfiera de modo temporal o permanente en los procesos evolutivos de los niños. Los hijos se ven expuestos a mayor estrés, el cual puede constituir un factor de riesgo para la manifestación de dificultades en el logro de tareas evolutivas importantes, tales como un adecuado proceso de socialización, de desempeño escolar y de desarrollo socioafectivo.

La separación de los padres constituye una crisis dentro del ciclo vital de una familia. Esta crisis implica el rompimiento de la estabilidad y la estructura familiar previa, impulsando a la familia a que experimente un proceso de reajuste estructural y funcional significativos (Slaikeu, 1996).

Desde una descripción sistémica de los procesos que ocurren durante la separación, se considera que frente a esta crisis los límites del sistema familiar se aflojan y aumenta la permeabilidad, con lo cual el intercambio de información se incrementa entre la familia y su contexto. A su vez, las reglas de con-

1 En el presente artículo se utiliza el género masculino para referirse a los niños en general, e incluye las opiniones tanto de los niños como de las niñas que participaron en la investigación. vivencia y los roles de los miembros se confunden, promoviendo así la sensación de inestabilidad; las expectativas y las prohibiciones se relajan, las metas y valores pierden importancia y pueden incluso desaparecer, se reviven conflictos irresueltos, aumenta la tensión entre los miembros, se generan sentimientos de desorientación y se dificulta el proceso de toma de decisiones (Gianella \& Pérez, 2004).

De modo global, la respuesta que los sistemas familiares habitualmente manifiestan frente a esta crisis suele ser de dos tipos. Una en la que la familia logra mantener las funciones de protección hacia los hijos/as, y otra en la que se mantiene vigente el conflicto de la pareja que se separa, en desmedro de las funciones parentales de cuidado (Cáceres, Manhey \& Raies, 2004).

Isaacs, Montalvo y Abelsohn (2001) señalan que, frente a la separación de los padres, tanto la pareja como los hijos deben modificar sus relaciones con las redes sociales, propias y compartidas, estableciendo alianzas y nuevas coaliciones. A menudo, la familia debe adaptarse a un cambio radical en su posición socioeconómica, lo cual altera su estilo de vida. Según estos mismos autores, la mayoría de las parejas que se separan tienden a proteger a sus hijos/as del conflicto entre los adultos y de la desorganización de la vida familiar, sin embargo, hay ocasiones en que esto resulta muy difícil. En este sentido, se habla de divorcios difíciles, para referirse a dinámicas tales como las que protagonizan padres que mantienen sus disputas y hacen que los hijos tomen partido en el conflicto. Otro de los escenarios posibles se da cuando los adultos pierden la confianza en su capacidad para cumplir las tareas parentales, o bien abdican de su responsabilidad en la educación y crianza de los hijos. De acuerdo con estos investigadores, son en definitiva estas dinámicas las que más interfieren en el proceso del niño respecto de la elaboración de la crisis de la separación.

\section{Separación de los padres: iun evento dentro de la historia familiar o una experiencia traumática?}

A partir de una comprensión sistémico-relacional del proceso de separación, Cáceres et al. (2004) 
señalan que una representación traumática de la experiencia de separación interfiere en el proceso madurativo de los hijos/as. Esto se puede observar en diversas áreas del desarrollo, tales como la aparición de problemas afectivos, de dificultades de adaptación y en el empobrecimiento de sus relaciones sociales.

Cuando una crisis es vivida como trauma, de acuerdo con lo que postula Cyrulnik (2003), esta experiencia es significada por la persona como un evento que estanca el desarrollo personal y familiar. A partir de entonces, el sujeto percibe que su vida se ve partida en dos, dividida en un antes y un después del evento traumático. Respecto del concepto de trauma, Cyrulnik (2001) plantea que son dos los golpes que le otorgan la característica de trauma a un evento. El primero de ellos tiene lugar en la realidad y se vincula al dolor del daño o de la pérdida que sufre el sujeto. El segundo golpe se produce en la representación que el sujeto realiza de esta realidad, el significado que le otorga a lo sucedido en su historia personal, en el contexto familiar y social que lo rodea. Según este autor, es en el segundo golpe donde el dolor se transforma en un sufrimiento que acompaña a quien lo padece, y en torno al cual gira su existencia y se forja su identidad.

La separación de los padres se torna en un evento relevante para la vida de los niños a partir de dos dinámicas: el silenciamiento y el ser testigo del conflicto persistente entre los padres (Cáceres et al., 2004). La dinámica del silenciamiento surge del deseo de uno o ambos padres de superar esta crisis rápidamente. El temor de los padres por haber causado daño a los hijos/as dificulta la elaboración de la experiencia en el lenguaje. Los padres y los niños callan y así alimentan la fantasía de que la experiencia de separación no tuvo consecuencias adversas para los miembros de la familia (Cáceres et al., 2004). De este modo, el silenciamiento impide que los niños construyan narrativas compartidas con los demás miembros de la familia acerca de los acontecimientos, lo cual contribuiría a legitimar la experiencia vivida. La posibilidad de elaborar narrativas no traumatizantes sobre la experiencia dolorosa, como señala Cyrulnik (2003), permite proteger y dinamizar los recursos de las personas que han vivido crisis significativas.

La otra alternativa a través de la cual la experiencia de la separación de los padres se vuelve un evento traumático para los niños corresponde a la dinámica de conflicto intenso y sostenido que se produce entre los padres, aún después de producida la separación. Esta experiencia afecta negativamente a los hijo/os quienes a menudo se ven envueltos en las peleas de la expareja. En consecuencia, los niños manifiestan, a través de conductas y síntomas, la tensión permanente en la que viven. En estos casos, la familia no se puede reorganizar funcionalmente, lo hace en torno al conflicto. De este modo, ni los padres ni los hijos logran reconstruir una identidad que vaya más allá de la experiencia de separación (Cáceres et al., 2004). En estas familias, indican los autores, la separación pasa a ser el núcleo en torno al cual organizan su existencia.

\section{Consecuencias de la separación para los niños}

Para los niños que han vivido inicialmente con ambos padres, este resulta ser el escenario habitual donde se desarrollan y desenvuelven sus actividades cotidianas. Lo ajeno, lo inesperado es situarse en una convivencia donde uno de los padres ya no vive en el hogar. Este hecho genera un quiebre en la noción que el niño tiene de su realidad familiar exponiéndolo al estrés de tener que enfrentar cambios significativos en sus rutinas de vida.

Según Jongsma, Peterson y McInnis (2000), las consecuencias de esta crisis en los niños suelen ser: contacto insuficiente o pérdida de contacto con una de las figuras paternas luego de la separación o el divorcio; pérdida de contacto con redes de apoyo significativas debido a cambios de casa o ciudad; reacciones emocionales intensas de llanto y queja; miedo y preocupación respecto de ser abandonados o separados de sus padres; sentimientos de culpa por la separación de los padres; sentimientos de dolor y tristeza combinados con baja valoración personal, desconfianza, inseguridad y/o pérdida del interés en actividades que antes les generaban placer; aumento en la frecuencia de problemas de conducta; desmo- 
tivación escolar y baja en el desempeño; conductas regresivas; manifestaciones de seudo madurez -asumir roles parentales y nuevas responsabilidades- y las quejas psicosomáticas.

De acuerdo con Wallerstein, Lewis y Blakeslee (2001), las tareas psicológicas que deben enfrentar los niños con la separación de los padres son: lograr comprender en forma realista lo que la separación de sus padres implica para la familia y cuáles serán los cambios concretos que deberán asumir; la retirada estratégica de los hijos del conflicto parental; la pérdida de la familia intacta y la pérdida de la percepción, simbólica o real, que se tiene respecto de la protección que la familia le ha brindado; además, deben asumir la ausencia de uno de los padres de modo cotidiano en el hogar, usualmente el padre y que la separación de los padres es una decisión voluntaria de al menos uno de ellos; enfrentar el dilema que implica reconocer que todos estos cambios familiares han sido provocados por las personas a cargo de su protección y crianza; trabajar los sentimientos de culpa frente a la separación de los padres; aceptar que la separación de sus padres es permanente y recuperar la confianza en el amor. Así como lo plantea Roizblatt (1997, p. 11):

La positiva adaptación de los niños a la separación se relaciona con la capacidad de los padres para limitar la separación a la pareja y no a los hijos; evitar los conflictos de lealtades y mantener su estabilidad, dando oportunidad a los hijos para compartir sus problemas relacionados con la separación de sus padres, con sus amigos y personas más cercanas; que los hijos tengan la posibilidad de mantener una relación cercana y constante con ambos padres; además de tener la posibilidad de una adecuada preparación previa a la separación.

\section{Elementos que contribuyen a la elaboración de la crisis por parte de los niños}

Como toda experiencia difícil, la separación de los padres debe ser abordada de la mejor manera posible para así aminorar su impacto en el desempeño habitual de los niños. En consecuencia, los estudios de Garmezy (1983 citado en Grych \& Fincham,
1997) destacan como factores protectores de los niños en situaciones de estrés, variables como una autoestima positiva y el contar con apoyo familiar, destacando la participación de la familia extensa y la intervención de organizaciones sociales, principalmente de la escuela. En este sentido, las personas que conforman la red de apoyo social del niño cumplen roles importantes en actividades tales como la recreación, el dar consejo, el otorgar ayuda en tareas cotidianas, ser fuente de apoyo emocional y/o proveer de retroalimentación positiva (Wolchik et al., 1989 citados en Grych \& Fincham, 1997).

A su vez, Leon (2003) destaca el adecuado monitoreo parental y la participación de los padres en las actividades de sus hijos/as. Y, por el contrario, el verse involucrado en los conflictos de lealtades entre los padres, constituye uno de los factores de mayor riesgo para los niños a edades tempranas.

En situaciones de crisis, se espera que los padres den a sus hijos/as el apoyo que necesitan, sin embargo, dada la presión y el estrés psicológico y emocional que les demanda la crisis de pareja, a menudo estos no están ni emocional ni físicamente disponibles para atender a las necesidades de sus hijos/as. En este sentido, la posibilidad de contar con otros vínculos afectivos y de confianza resulta fundamental para enfrentar y sobrevivir a situaciones adversas. Los procesos relacionales son relevantes en la medida en que dan lugar a una construcción colectiva de narrativas coherentes, en las que los dolores y las adversidades tienen un sentido comprendido y compartido por todos, dignificando esas vivencias, y movilizando así los recursos de quienes las sufren Ravazzola (2001).

Cyrulnik (2003), por su parte, emplea el concepto de tutores de resiliencia, para destacar la importancia de redes de apoyo que entreguen un soporte afectivo y contribuyan a otorgarle un sentido a la experiencia vivida. Para este autor, el que una experiencia traumática se constituya en un aspecto vital integrado a la historia del sujeto, depende en gran medida de la representación que este realice de este evento; de ahí la relevancia de considerar la presencia de tutores afectivos que promuevan en los niños la construcción de narrativas no traumatizantes que le den sentido a sus experiencias. 


\section{Método}

\section{Características de la muestra}

La estrategia de muestreo utilizada consideró los procedimientos del muestreo teórico, descrito por Glaser y Strauss (1967). En consecuencia, se procedió a seleccionar a los primeros sujetos y se analizaron los datos obtenidos. Mediante el análisis de estos primeros datos se desarrollaron conceptos, categorías conceptuales e hipótesis que fueron utilizadas para generar criterios mediante los cuales se seleccionó a los siguientes sujetos que se integraron a la muestra. Los primeros sujetos fueron elegidos con base en el conocimiento teórico y/o práctico previo, existiendo un marco de referencia inicial de selección de la muestra delimitado por el problema de estudio (Krause, Cornejo \& Radovcic, 1998).

El número de entrevistados estuvo sujeto al logro de la "saturación teórica" de las categorías conceptuales y de las relaciones entre estas, generadas a través del análisis, es decir, hasta el momento en que los nuevos datos no agreguen información nueva (Krause et al., 1998). Por lo tanto, la muestra estuvo finalmente constituida por un total de 12 niños y niñas de edades entre los 9 y 12 años, en su mayoría de edades entre los 10 y los 11 años.

Todos eran hijos de padres separados hacía 2 o 3 años y pertenecían a un nivel socioeconómico medio, lo cual fue identificado a partir de la aplicación de un cuestionario adaptado por Ramírez y $\mathrm{Haz}(2001)^{2}$.

Como criterios de exclusión de la muestra, se consideraron aquellos niños que se encontraban asistiendo a procesos psicoterapéuticos y aquellos que estuviesen experimentando el proceso de reestructuración como familia simultánea.

De este modo, las distancias de las unidades muestrales se minimizaron y la comparación de los datos permitió verificar la utilidad de una categoría, visualizar aspectos antes no vistos, además

2 La Escala de Nivel Socioeconómico ha sido construida con base en varias escalas: la Escala Graffar adaptada en 1993 por Ramírez y Haz, la Ficha CAS-2 de las Municipalidades y otras fuentes que analizan dichas escalas. de ayudar a establecer el conjunto de condiciones bajo las cuales una categoría aparecerá en un grupo determinado y a establecer las propiedades de una categoría (Krause et al., 1998).

\section{Diseño y procedimiento}

Este estudio posee un diseño descriptivo relacional y se empleó una metodología cualitativa. Esta elección se basa en las características del objeto de estudio, el cual corresponde a las narrativas infantiles respecto a la separación de los padres y el significado que le atribuyen a esta experiencia. De acuerdo con Patton (1980), la metodología cualitativa resulta el método más indicado para el estudio de las perspectivas de otros, cuyos procesos o fenómenos no podemos observar directamente y/o sobre los cuales no se tienen antecedentes teóricos y empíricos previos. En consecuencia, para efectos de este estudio, dicha metodología es coherente con el objeto de estudio preliminar, ya que permite producir datos descriptivos con base en un conjunto de métodos y procedimientos, privilegiando la información proveniente desde la perspectiva de los niños. Además, este método, de acuerdo a los procedimientos de la escuela Grounded Theory, permitirá tanto la generación de resultados descriptivos como la creación de modelos teóricos sobre el problema en estudio (Glaser $\&$ Strauss, 1967).

Como primer paso, se efectuó un focus group con expertos del área de la Psicología Infantil y Psicología Educacional con el objetivo de registrar información relevante que aportara a la elaboración del guión de entrevista semiestructurada que se emplearía con los niños. Luego, se procedió a la construcción del guión de entrevista y a la selección de los primeros entrevistados, considerando los criterios de muestra antes mencionados.

El acceso a la muestra implicó una fase inicial de identificación de casos para lo cual se recurrió a la colaboración de diversos establecimientos educacionales de Santiago, Concepción y Valparaíso. Estas tres ciudades fueron elegidas con un criterio de conveniencia, ya que se tenía un contacto pre- 
vio con estos colegios lo cual facilitó el acceso a la muestra.

Como estrategia, se hizo llegar una carta de presentación del estudio a los directores de colegios. Una vez registrado el interés por participar previa autorización y colaboración de los directores de establecimientos educacionales, se identificaron los casos y se precedió a entrevistar a los apoderados con el fin de informales respecto del estudio y de su participación en la investigación. Una vez obtenida la autorización del apoderado, se procedió a registrar antecedentes familiares generales correspondientes a la historia de separación de los padres con el objetivo de contar con directrices para la entrevista con los niños. Adicionalmente al conocimiento de las madres, se les aplicó un cuestionario de nivel socioeconómico de Ramírez y Haz (2001).

Luego de la entrevista con la madre, se citó al niño a una entrevista individual de 60 minutos de duración. Previo al inicio de la entrevista, se solicitó la autorización individual de cada niño en el estudio. Los datos fueron grabados en cinta de audio, transcritos a formato de texto escrito, codificados y analizados por el investigador. Luego de las primeras entrevistas, el guión fue dirigido por la teoría emergente, siendo factible efectuar preguntas más directas en relación a las categorías que surgieron de los datos.

La recolección de datos se realizó de acuerdo con el diseño de la investigación y se desarrolló de modo simultáneo con el análisis, siendo esta recolección flexible para adecuarse a lo que en cada etapa del estudio se deseaba saber. Así, al comienzo del estudio se realizaron entrevistas semiestructuradas a niños, las cuales se basaron en un guión predeterminado de preguntas generales, para luego profundizar a partir de la información expresada por los sujetos y así conocer su perspectiva acerca del tema (Fontana \& Frey, 1995). Luego de finalizada la entrevista con los niños se sostuvo una entrevista de devolución con sus respectivas madres, para proporcionarles información respecto del desempeño de su hijo en la entrevista y señalar directrices para el abordaje de temas asociados a la separación.

El análisis de los datos se desarrolló de acuerdo a los procedimientos consignados por la Grounded Theory (Glaser \& Strauss, 1967). Esto implica que, una vez aplicadas y transcritas, las entrevistas sean categorizadas dando origen a un primer bosquejo del sistema de códigos, el que es corregido tanto en forma como en contenido. Luego, se procede a codificar toda la información entregada por las entrevistas, de acuerdo al sistema antes desarrollado. Se efectuaron las correspondientes codificaciones: abierta, axial y selectiva. La primera, consiste en un proceso mediante el cual se fragmenta, examina, compara, conceptualiza y categoriza la información de manera descriptiva. Las siguientes, comprenden a un conjunto de procedimientos mediante los cuales la información que ha sido fragmentada en la primera etapa, se vuelve a ligar, utilizando para ello un paradigma de codificación (Glaser \& Strauss, 1967).

De este modo, la codificación axial permite establecer conexiones e interacciones entre categorías y subcategorías, para luego desarrollar, por medio de la codificación selectiva, la categoría central. De esta forma, se pretende obtener un modelo teórico descriptivo y relacional de la temática en estudio. Para la presentación de los resultados, se describirá cada una de las categorías encontradas y la organización de los resultados en áreas temáticas.

\section{Resultados}

\section{Análisis relacional del modelo}

En la siguiente figura se muestran las variables estudiadas en relación al fenómeno de la separación de los padres y cómo evoluciona el proceso de asimilación de la experiencia desde la perspectiva infantil.

¿Cuáles son las creencias, los sentimientos e ideas de los niños respecto de la separación?

$\mathrm{Al}$ analizar las narrativas infantiles podemos observar que cada niño posee un registro particular y claro de aquellos aspectos más significativos de esta vivencia. Para los niños entrevistados, la separación de los padres constituye una crisis que impacta sus vidas introduciendo cambios en la organización familiar, lo cual coincide con lo plantado por los enfoques teóricos de análisis sistémico de la experiencia. 


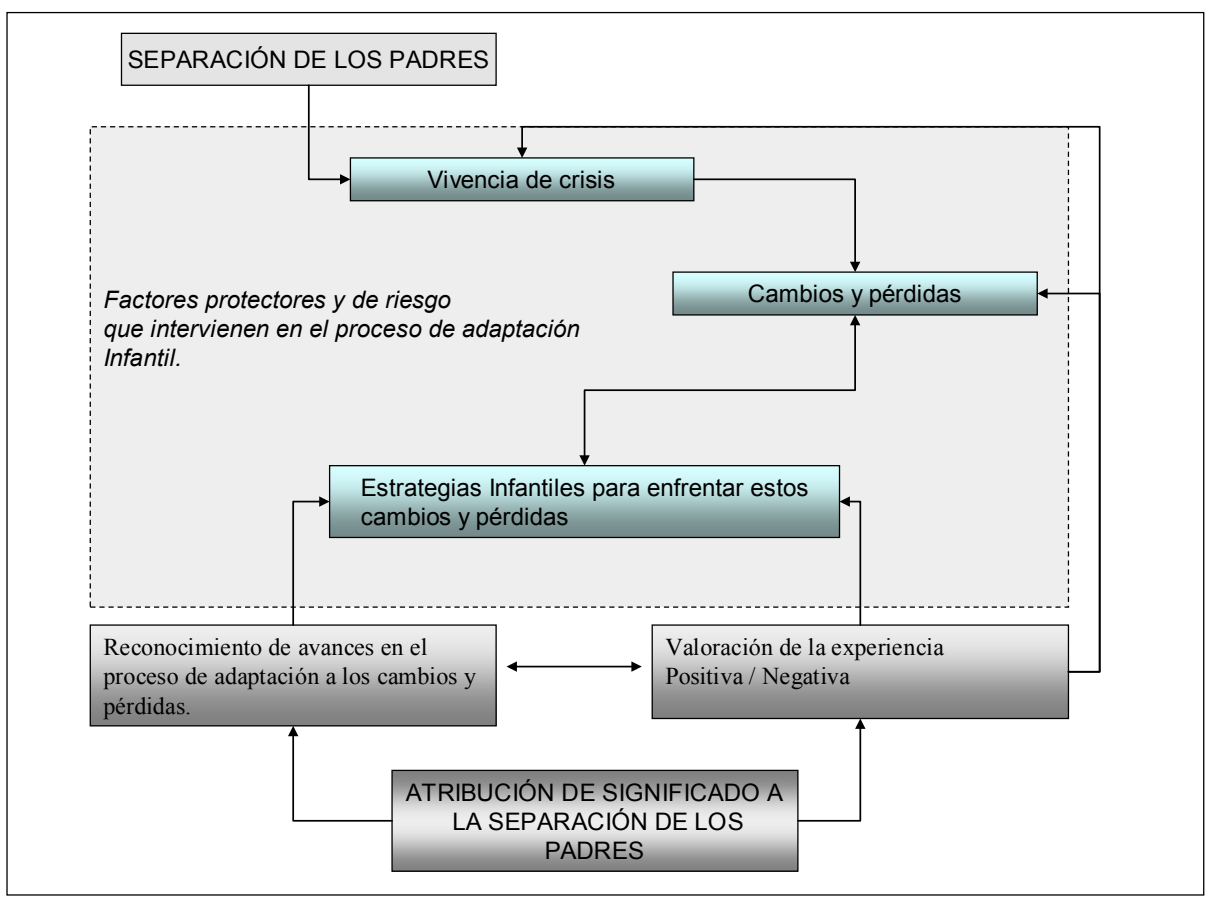

Figura 1. Atribución de significado a la experiencia.

Fuente: Cifuentes (2007).

Esta crisis se evidencia en los relatos en detalles tales como: quién les dio la noticia de la separación; cuáles eran las características contextuales del momento en que se les informó del hecho; qué argumentos se le dieron para comprender la decisión tomada por sus padres; y cuáles son las reacciones inmediatas de los demás miembros de la familia. Ciertamente, no da lo mismo quién informa al niño de la separación y en qué condiciones emocionales se encontraba al momento de transmitir la noticia.

E.-iTú lo alcanzaste a ver cuando se estaba yendo? N.-Sí y me dijo: "Quiero que cuides a tu hermano, quiero que cuides harto a tu hermano y que te cuides tú"; y me dijo: "Chao".

(VII: 167-168, 10 años)

Como se puede apreciar en esta cita, la información que reciben los niños, difícilmente es la necesaria para comprender con claridad lo que significa esta experiencia. En consecuencia, lo que el padre o la madre digan o dejen de decir a sus hijos/as respecto de la separación, influirá de manera significativa en la asimilación de la crisis y en la construcción de narrativas que les otorguen sentido a esta experiencia.

Otros de los aspectos que se debe tener en consideración son los argumentos que los adultos les brindan a los niños. En los casos entrevistados, los argumentos más frecuentes de la separación fueron los conflictos entre la pareja y la infidelidad, siendo este último el que mayores confusiones traía a la vivencia infantil.

E.-... Y iqué crees que ha sido como lo más difícil del proceso después de la separación de tus papás?

N.-Em...

E.-Como de los cambios...

N.-Acostumbrarme a no estar con mi papá, porque... yo era así como muy apegada a mi papá y... aceptar así que... aceptar que mi papá ya no iba a volver y que tenía otra.

(III: 294-302, 10 años)

Lo complejo de estos argumentos es que los niños a su corta edad, no cuentan con las estructuras 
cognitivas que les permitan comprender lo que implica una infidelidad y, en consecuencia, se exponen a conflictos mayores, ya que los padres están atrapados en sus sentimientos de rabia y traición. Tal como se discutió en el focus group realizado al inicio del estudio, la infidelidad constituye uno de los argumentos que más perturba la elaboración de la experiencia por parte de los hijos.

Continuando con los elementos que se destacan de la narrativa infantil, se encontró que las reacciones emocionales de los demás miembros de la familia impactan a los niños. El haber visto al padre o a la madre llorar genera en los niños sentimientos de pena que los llevan a solidarizar con ellos, estableciéndose alianzas que pueden derivar en conflictos posteriores de lealtad.

Las reacciones emocionales de llanto que manifiestan los padres frente a los hijos/as, son interpretadas por los niños como la vulnerabilidad del adulto y, en consecuencia, poco disponibles para responder a las necesidades afectivas de los niños, lo cual incrementa el estado de confusión y angustia, propios de la crisis. Frente a esta situación, los hijos suelen ocultar sus expresiones de pena y rabia frente a sus padres y recurrirán a otras redes de apoyo, ya sea dentro o fuera del contexto familiar.

El primer indicador que marca el inicio de la crisis para los niños, lo constituye la partida del padre del hogar. Este episodio queda en la memoria de los niños señalando el comienzo de un proceso de ajuste. Los niños extrañan la participación del padre en sus actividades escolares, en los momentos de juego e incluso, su sola presencia en el hogar, no importándoles el tener una interacción lúdica con él, tal como lo sostiene el siguiente párrafo:

E.-¿Qué es lo que ha sido lo más difícil en este proceso de cambios, de adaptarse a vivir en dos casas?

N.-Mmm... yo creo que no estar con mi... otro familiar porque antes yo... siempre jugábamos los tres, cosas, bachillerato, juegos de mesa y ahora puedo jugar, no puedo jugar con mi mamá porque yo siento que nos falta uno... en la familia... nos falta uno.

(II: 128-129, 11 años)
Este aspecto es relevante ya que manifiesta la sensibilidad con que los niños resienten el cambio de escenario familiar y cómo rescatan y valoran hasta el más mínimo detalle que les permite confirmar la presencia del padre en sus vidas. En consecuencia, es importante reconocer esta vivencia y respetar el período de ajuste y duelo que sufren los niños con la partida del padre. Dar espacios para sentir y expresar estas sutilezas que van marcando los cambios y pérdidas, y el surgimiento de una nueva organización familiar para los hijos. Como consecuencia de los cambios, resulta relevante poder garantizar la continuidad de un vínculo paterno saludable para los niños, a fin de atenuar las consecuencias emocionales de su ausencia.

Otros tipos de cambios que comentan los niños tienen que ver con el mudarse de casa, la inserción a un nuevo grupo familiar -como es el de los abuelos- y/o cambios de colegio, considerando las pérdidas de amistades que esto implica. En este ámbito, aparecen los ajustes en las tareas y rutinas en el hogar, como por ejemplo, quién los lleva al colegio, quién participa de sus cumpleaños y los horarios de visitas. Otros cambios se relacionan con que los niños ayuden en las tareas domésticas y su adaptación a la salida de la madre del hogar por su inserción en el mundo laboral. El trabajo materno fuera del hogar genera sentimientos confusos en los niños, ya que, por una parte, comprenden que debe trabajar y, por otra, la perciben como menos disponible para ayudarlos en tareas escolares y juegos, incrementándose los sentimientos de soledad durante las primeras etapas del proceso de separación.

E.-¿Por qué crees tú que a los niños les hace mal que sus papás se separen?

N.-Porque, digamos, cuando los papás se separan y más que nada por ejemplo que la mamá se quede con el niño, tiene que trabajar para mantener la familia, no. Entonces no pasan tanto tiempo, entonces... digamos, cuando el niño necesita que le ayuden en una tarea eee, no no tienen el tiempo y se sienten mal. Digamos, cuando tienen que llevar materiales y tu mamá no te los puede comprar a si... por ejemplo. O tú te sientes mal 
porque todas las mamás van a llevar los materiales... y todo eso que te piden y en cambio tú, te vas a quedar sola, con... haciendo nada.

E.-Eso es como medio injusto entonces.

N.-Sí... porque al final los que quedan pagando son los niños que no hacen nada.

(V: 183-186, 9 años)

La reacción emocional inicial que se registra con mayor frecuencia en los casos entrevistados tiene que ver con sentimientos de pena, rabia, frustración. Habitualmente, los niños recuerdan haber llorado luego de haber recibido la noticia. Luego de estas primeras respuestas, los niños suelen asumir diversas acciones a nivel individual y colectivo a fin de enfrentar el evento.

En este sentido, lo más destacable es la presencia de redes de apoyo familiar y social que contribuyen a la asimilación de la crisis. Los niños destacan el apoyo recibido de sus amigos, familiares y profesores, reconociendo la importancia de tener alguien con quien conversar y compartir su experiencia.

N.-O sea, igual a la primera que le conté fue a mi mejor amiga pero que ella no dijo ni pío porque nos tenemos harta confianza.

E.-Ella te mantuvo el secreto.

N.-Hasta el día que le conté a otras personas, a mi profesor también... porque en kinder él me empezó a ayudar entonces me ayudaba en las pruebas, en las tareas.

E.-Y estudiaban juntos.

N.-Sí.

E.-Y... en ese sentido, el poder hablarlo con otras personas, que fuera tu amiga o tu profesor... ite sirvió?

N.-Em... sí porque como que... cada vez que lo contaba me iba sacando más pena, entonces igual no por contarlo a todo el colegio me iba a alegrar, sino que era como un $0.1 \%$ lo que me sacaba.

E.-Y ite aliviaba ese cero coma algo que te sacabas de encima?

N.-O sea... me aliviaba decírselo porque... me sacaba un silencio que tenía guardado hace harto tiempo igual, cinco meses, seis meses... Hay hartos compañeros que están separados... Por ejemplo el Leonardo, la Montserrat y todo eso. (I: 316-324, 12 años)

Estas personas que acompañaron y apoyaron a los niños en un momento fundamental de sus vidas, pasan a tener un lugar significativo en el recuerdo y en el vínculo emocional que los niños sostienen con ellas. Son estas oportunidades de relación afectiva, no enjuiciadora, las que marcan una diferencia en la manera en que los niños relatan la experiencia vivida $y$, en consecuencia, en la manera en que representan los hechos en el recuerdo. Contar con una red de apoyo social y familiar disponible constituye uno de los factores protectores más relevantes descritos por la literatura.

Sin embargo, también es posible reconocer junto con la presencia de estos factores protectores, dinámicas que constituyen factores de riesgo para la elaboración de la crisis, en la medida en que sobreexigen a los niños al imponerles responsabilidades que exceden sus capacidades. Un ejemplo de este tipo de riesgos es la parentalización, donde los hijos mayores deben asumir el cuidado de los más pequeños.

E.-Tienes hartos hermanos... ¿cómo es esto de tener más hermanos y ser el tercero de una familia de 4 ?

N.-no sé... como que... yo igual tengo responsabilidades porque mi hermana no está mucho en la casa... porque tiene que hacer unos exámenes... porque está embarazada... y mi mamá la acompaña y tiene que salir a trabajar... y yo soy el que me quedo... a cargo de los más chicos... entonces yo me tengo que preocupar de que tomen onces cuando no esta mi mamá... de todo eso... que si van a salir que estén por ahí cerca de la casa... y cuando llega mi mamá de trabajar ahí yo descanso un rato... (VIII: 47-48, 12 años, 12 años)

La imposición de los mandatos paternos respecto del cuidado de los hermanos menores y la delegación de responsabilidades adultas en los hijos mayores, producto de la nueva organización fami- 
liar, suelen provocar dinámicas de parentalización que hacen que los niños asuman roles que no les corresponden, y que les dejan poca energía emocional y psicológica para identificar y satisfacer sus propias necesidades afectivas.

Sin embargo, pese a lo demandante que pudiese ser el asumir un rol de adulto frente a los hermanos menores, el factor que más parece afectar la elaboración de la experiencia de separación de los padres tiene que ver con la pérdida definitiva del vínculo con la figura paterna. Este factor interfiere de modo significativo en la manera en que los niños enfrentan la crisis y se explican los hechos.

E.-Y respecto a tu papá una vez que se separaron... ¿cómo fueron las visitas?... ¿lo pudiste seguir viendo...?

N.-Cuando se separaron al principio me venía a ver. Pero ahora ya no me viene a ver. (IX: 112-113, 11 años)

La pérdida del vínculo con el padre constituye uno de los elementos que más interfiere en el proceso de elaboración de la experiencia. Aun cuando la relación con el padre y la dinámica familiar previa a la separación hayan estado teñidas por el conflicto, los niños añoran la presencia del padre en sus vidas, más aún, en aquellos casos donde la relación padre-hijo era percibida como nutritiva desde la perspectiva de los niños. De ahí que las narrativas infantiles destaquen el contar con las visitas regulares del padre/madre, como elemento que facilita la integración de la experiencia.

Parte del proceso reflexivo que hacen los niños al recodar su experiencia da cuenta de la valoración de los hechos y el reconocimiento de sus propios desempeños frente a la situación de crisis sufrida. Los niños entrevistados reconocen indicadores concretos de estos cambios, por ejemplo, el haber recuperado el interés por sus actividades recreativas; la disminución de sentimientos de pena, rabia y/o vergüenza asociados a la separación y el incremento en el interés por las responsabilidades escolares.

Junto con el reconocimiento de avances, los niños analizan los antecedentes y establecen una valoración de su vivencia. Dentro de los aspectos que se consideran en esta valoración están los cambios y pérdidas sufridos a causa de la separación; el reconocimiento de aquellos aspectos más difíciles de enfrentar durante la separación; la identificación de redes de apoyo; el registro del nivel de conflicto familiar previo y posterior a la separación; y, por último, la presencia o ausencia del padre luego de la separación. De todos estos elementos los más significativos fueron la existencia de redes de apoyo, el registro del nivel de conflicto existente en el hogar y la continuidad del vínculo paterno.

La valoración negativa de la experiencia esta dada por el quiebre de la representación de familia nuclear, constituida por los padres y los hijos. Los niños que no contaron con la presencia del padre luego de la separación, solían presentar más sentimientos de soledad y culpa por la separación, así como también eran más frecuentes las quejas y el cuestionamiento a los argumentos adultos. En aquellos casos donde sí existía presencia paterna después de la separación, pero en los cuales el conflicto entre la expareja continuó -exponiendo a los hijos a conflictos de lealtades entre los padres- también se cuestionan los argumentos de la separación.

La valoración positiva de la experiencia de tener padres separados esta relacionada con la capacidad de los niños de resignificar los cambios en términos más constructivos, lo cual implica reconocer aspectos favorables de la separación, tales como la disminución del nivel de conflictos al interior de hogar; las mejoras en la relación entre hermanos, el incremento de los actividades recreativas que realizan con los padres y la continuidad del vínculo paterno.

A partir de este proceso de registro, identificación y análisis de los hechos vividos, los niños representan su experiencia, integrando cada elemento de manera coherente en sus narrativas. Estos relatos, compartidos luego de transcurridos 2 o 3 años de la separación de los padres, evidencian la capacidad de toma de perspectiva de los protagonistas respecto de la experiencia, quienes son capaces de transmitir un discurso rico en vivencias y detalles que comunican la complejidad e intensidad de los hechos vividos. 


\section{Conclusión}

La narrativa infantil sobre la experiencia de la separación de los padres, da cuenta de una vivencia de crisis que se evidencia en las características de un relato rico en detalles de momentos previos y posteriores a la separación, señalándose con claridad los argumentos y las reacciones personales y de los demás integrantes de la familia frente a la noticia. Los niños recuerdan aspectos relevantes de la convivencia familiar previa que les hacían sospechar de una eventual separación, sin embargo, esta sospecha no les evita el impacto y la reacción de sorpresa al ser informados por sus padres.

Para los niños es fundamental recibir un mensaje honesto y esperanzador de parte de los adultos que expliciten temas como las visitas, el lugar donde vivirá el padre luego de la separación y si podrán reunirse en ese sitio con él. Los niños enfrentan la reestructuración de su ambiente familiar y, en consecuencia, les resulta profundamente aliviador que se les garantice la continuidad del vínculo con el padre, además del compromiso de término de conflictos en el hogar. Esto constituye el elemento diferenciador que permite alentar las fuerzas de los niños para hacer frente a la separación.

Otro elemento que afecta la valoración de la experiencia tiene que ver con el registro de conflictos y peleas entre los padres, aun después de la separación, incluso cuando han trascurrido dos o tres años de este episodio. Estas dinámicas de perpetuación del conflicto dificultan los procesos de asimilación y adaptación de los hijos al nuevo contexto familiar, en la medida en que se contradicen los argumentos aludidos por los propios padres para justificar la separación. Como consecuencia, los niños sienten que su dolor y sufrimiento no ha servido de nada, ya que han tenido que pasar por esta crisis sin vivenciar ningún resultado de cambios y/o mejoras en la convivencia familiar. Esto motiva una valoración negativa de la experiencia y además promueve las fantasías de reconciliación de los padres.

En relación a las redes de apoyo percibidas por los niños como recurso protector, se comprueba la eficacia de la presencia de vínculos con abuelos, hermanos mayores, compañeros de colegio y profesores. La relación entre pares se vuelve un referente que estimula a los niños a superar la crisis. El altruismo entre los niños constituye un recurso maravilloso que surge de la vivencia empática de haber experimentado el dolor y sufrimiento.

Sin duda, los niños entrevistados cuentan con recursos importantes, tales como autoestima positiva, capacidad de establecer vínculos, recursos socioemocionales para comunicar sus sentimientos y permitir ser contenidos y apoyados por otros, principalmente por sus pares. En términos generales, los niños perciben la separación como una experiencia negativa en sus vidas, sin embargo, la descripción y representación de los hechos no queda estancada en los recuerdos del dolor y las pérdidas sufridas, sino que va más allá, pudiéndose conocer cómo los niños vuelven a mirar su historia y se reconocen desde un presente distinto, donde pueden describir sus recursos e identificar su proceso de avance y superación de la crisis.

En definitiva, el relato de los niños está construido a partir de recuerdos emotivos, cargados de sentimientos confusos de rabia, pena y a veces vergüenza. A esta vivencia emocional se le deben sumar las interferencias de los mensajes contradictorios que los adultos transmiten, contribuyendo a generar mayor incertidumbre en los niños. Entonces, no debieran extrañarnos las dificultades que encuentran los niños para construir un relato coherente que articule debidamente toda la información que ellos han registrado de los hechos.

Las parejas se separan con la ilusión de acabar con sus conflictos y esto puede ser cierto; sin embargo, deben estar concientes de que su decisión no significa que la expareja desaparecerá de sus vidas y menos de la de sus hijos. Más bien, habrá que desarrollar nuevas maneras de relación que permitan comunicarse y negociar, a fin de posibilitar el surgimiento de una coparentalidad que garantice el desarrollo y bienestar de los niños. Se debe asumir que las negociaciones y planificaciones serán una constante en la vida de los hijos. Ello implica aprender a compartir los tiempos, definir con quién se pasa la Navidad, el Año Nuevo, los cumpleaños, la Primera Comunión, las graduaciones, a quién invitar a los actos del colegio, etc. Todas las fechas 
relevantes para la vida de los niños estarán precedidas del cuestionamiento y toma de decisión respecto de cuál de los padres deberá asistir.

Qué importante es entonces que los padres asuman su rol de cuidar y proteger a sus hijos, respetando las necesidades infantiles y dejando de lado los conflictos adultos, para así poder promover su desarrollo y bienestar. Estos actos de parentalidad competente y responsable contribuyen a superar la crisis de la separación, a resignificar la experiencia, a construir buenos recuerdos que nutran la memoria biográfica de los niños y aporten al desarrollo de su identidad, al darle un sentido que dignifica la experiencia sufrida.

\section{Referencias}

Cáceres, C., Manhey, C. \& Raies, A. (2004). Comprensión sistémico-relacional del proceso de separación conyugal. De Familias y Terapias, 18, 31-54.

Capponi, R. (2003). El amor después del amor. El Camino del amor sexual estable. Santiago: Grijalbo.

Cifuentes, O. (2007). Separación de los padres: competencias infantiles para enfrentar la crisis. Tesis no publicada de Psicología, Pontificia Universidad Católica de Chile.

Cyrulnik, B. (2001). Los patitos feos. La resiliencia: una infancia infeliz no determina la vida. Barcelona: Gedisa.

Cyrulnik, B. (2003). El murmullo de los fantasmas. Volver a la vida después de un trauma. Barcelona: Gedisa.

Fontana, A. \& Frey, J. (1995). Interviewing: The art of science. En N. Denzin \& Y. Lincoln (Eds.), Hanbook of qualitative research (pp. 273-285). Thousand Oaks: Sage Publications.

Gianella, C. \& Pérez, F. (2004, septiembre). Mediación, mediadores y familia. Ponencia presentada en el Seminario Mediación Familiar en el Contexto Judicial: Una Revisión de Experiencias, Facultad de Derecho y Facultad de Ciencias Sociales, Pontificia Universidad Católica de Chile, Santiago, Chile.

Glaser, B. G. \& Strauss, A. L. (1967). The discovery of Grounded Theory. Chicago: Publishing Company.
Grych, J. \& Fincham, F. (1997). Children's adaptation to divorce: From description to explanation. En S. A. Wolchik \& I. N. Sandler (Eds.), Handbook of children's coping with common stressors: Linking theory, research and interventions (pp. 159-193). New York: Plenum Press.

Isaacs, M., Montalvo, B. \& Abelsohn, D. (2001). Divorcio difícil: terapia para los hijos y la familia (2. - ed.). Buenos Aires: Amorrortu.

Jongsman, A. E., Peterson, L. M. \& McInnis, W. P. (2000). The child psychotherapy treatment planner

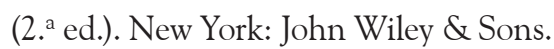

Krause, J., Cornejo, M. \& Radovcic, J. (1998). Diseño de estudios cualitativos (Proyecto de capacitación y asesoría para la realización de estudios cualitativos a equipos de alcohol y drogas). Santiago: Ministerio de Salud de Chile.

Leon, K. (2003). Risk and protective factors in young children's adjustment to parental divorce: A review of research. Family Relations, 52(3), 258-270.

Patton, M. (1980). Qualitative evaluation methods. Beverly Hills: Sage.

Ramírez, V. \& Haz, A. (2001). Escala de Nivel Socioeconómico. Manuscrito no publicado, Pontificia Universidad Católica de Chile.

Ravazzola, M. C. (2001). Resiliencias familiares. En A. Medillo \& E. N. Suárez Ojeda (Comps.), Resiliencia: descubriendo nuestras fortalezas (pp. 103-122). Buenos Aires: Paidós.

Roizblatt, A. (1997). La familia y la separación matrimonial. Santiago: Dolmen Ediciones.

Slaikeu, K. (1996). Intervención en crisis: manual para la práctica y la investigación (2a. ed.). México: Editorial El Manual Moderno.

Wallerstein, J. \& Blakeslee, S. (1996). Second chances: Men, women and children a decade after divorce. New York: Houghton Mifflin Company.

Wallerstein, J. \& Blakeslee, S. (2005). Y los niños iqué? Cómo guiar a los hijos antes, durante y después del divorcio. Bogotá: Grupo Editorial Norma.

Wallerstein, J., Lewis, J. \& Blakeslee, S. (2001). El inesperado legado del divorcio. Madrid: Editorial Atlántida. 